\title{
Splenectomy and distal pancreatectomy in advanced ovarian cancer
}

\author{
Eun Ji Lee", Soo Jin Park", Hee Seung Kim \\ Department of Obstetrics and Gynecology, Seoul National University College of Medicine, Seoul, Korea \\ Contributions: (I) Conception and design: EJ Lee, HS Kim; (II) Administrative support: HS Kim; (III) Provision of study materials or patients: HS \\ Kim; (IV) Collection and assembly of data: All authors; (V) Data analysis and interpretation: All authors; (VI) Manuscript writing: All authors; (VII) \\ Final approval of manuscript: All authors. \\ \#These authors contributed equally to this work. \\ Correspondence to: Hee Seung Kim, MD, PhD. Department of Obstetrics and Gynecology, Seoul National University College of Medicine, 101 \\ Daehak-Ro Jongno-Gu, Seoul 110-744, Korea. Email: bboddi0311@gmail.com.
}

\begin{abstract}
Splenectomy or distal pancreatectomy (DP) is sometimes performed for optimal cytoreduction in advanced ovarian cancer (AOC). In particular, it is considered to remove tumors involving the splenic hilum or the capsule of the spleen to secure tumor-free margins sufficiently. For splenectomy, the gastro-splenic ligament is opened, and the short gastric vessels are dissected. After the splenocolic ligament and splenic flexure of the colon are transected, the peritoneal attachments, including the splenorenal and splenophrenic ligaments, are divided to mobilize the spleen, and then the splenic artery and vein are identified and ligated separately. If DP is needed for en bloc resection of tumors, a linear cutting stapler is used to remove the tail of the pancreas, and suture reinforcement with 2-0 or 3-0 prolene on the cut section of the pancreas is performed to prevent postoperative pancreatic fistula (POPF). Immunization with a polyvalent pneumococcal vaccine is required after splenectomy to avoid overwhelming post-splenectomy infection (OPSI) caused by Streptococcus pneumoniae, Neisseria meningitidis, and Haemophilus influenzae. If POPF occurs after splenectomy or DP, continued drainage with close monitoring is needed with the administration of board spectrum antibiotics in grade A or B POPF according to the criteria of the International Study Group of Pancreatic Fistula (ISGPF). In contrast, grade C POPF requires aggressive management using nothing by mouth, total parenteral nutrition, and somatostatin analogs, and sometimes reoperation if deteriorating signs such as sepsis and organ dysfunction. Thus, the effort for preserving pancreatic tail is needed to reduce hospitalization and the risk of POPF despite the minimal impact of DP on the success rate of optimal cytoreduction.
\end{abstract}

Koywords! Ovarian cancer; splenectomy; distal pancreatectomy (DP)

Submitted Feb 17, 2020. Accepted for publication Apr 24, 2020.

doi: 10.21037/gs-2019-ursoc-09

View this article at: http://dx.doi.org/10.21037/gs-2019-ursoc-09

\section{Introduction}

Optimal cytoreduction is one of the most important prognostic factors in AOC. Although aggressive debulking surgery is required to minimize the size of residual tumor, upper abdominal surgery, including splenectomy or distal pancreatectomy (DP), is not always achieved in gynecologic oncologists (1), and $31-49 \%$ of them perform it with general surgeons (2).

In general, splenic metastasis is found in only $2.3-7.1 \%$ of patients with AOC because of the following reasons (3). First, the splenic involvement may be low because the spleen is an immune organ with lymphoid tissues and lymphocytes that can kill tumor cells, and the splenic capsule could act as a shield, and its contractile properties might be involved by squeezing the tumor cells out (4). 

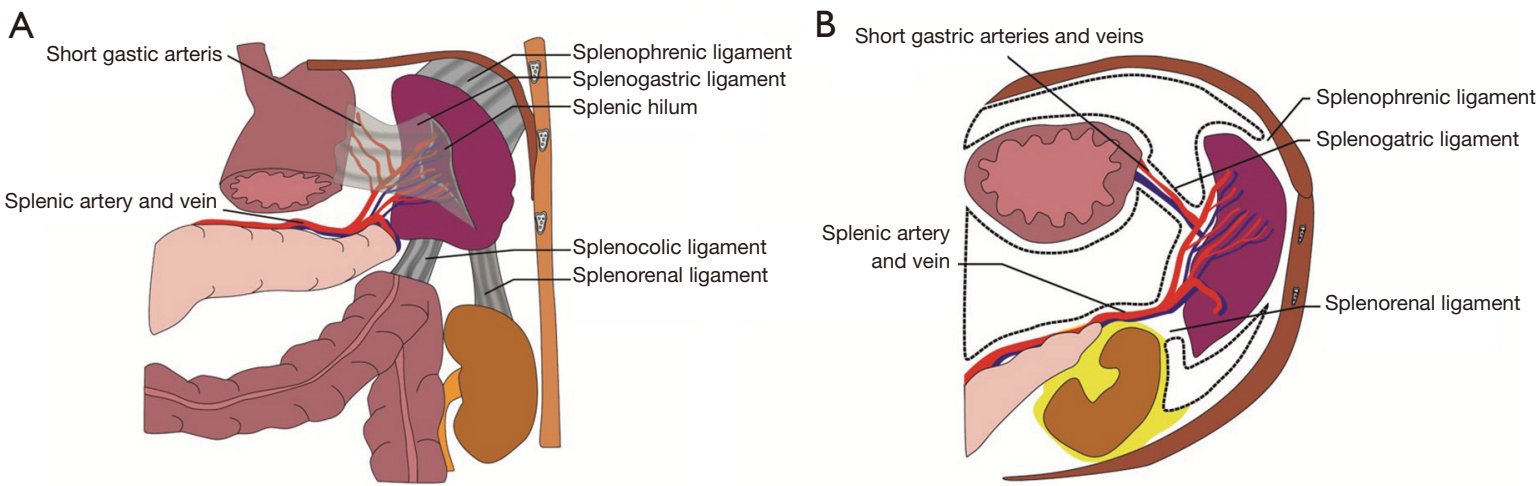

Figure 1 Ligaments that suspend the spleen: (A) coronal view; (B) transverse view.

Second, the tortuosity of the splenic vessels and abdominal fluid circulation could also contribute to the low rate of splenic metastasis (5). Moreover, only $6 \%$ of patients with AOC show pancreatic metastasis because of its rarity (6).

However, the tumor cells do not usually invade the splenic parenchyma. In contrast, the capsule of the spleen or the splenic flexure of the colon along with the gastro-colic ligament may be commonly involved, which occasionally requires to remove the intact spleen for removing the whole tumor mass (7), and thereby up to $31 \%$ of patients with AOC may receive splenectomy in the real world $(4,8-10)$. Moreover, the pancreatic tail is anatomically proximal to the spleen, and tumors invading the capsule of the spleen sometimes involve the splenic hilum in the patients. Thus, splenectomy for resecting tumors in the splenic hilum can be combined with DP to secure tumor-free margins sufficiently (11).

For gynecologic oncologists not familiar with splenectomy or DP, we will show the anatomy and operative procedure for splenectomy or DP, and relevant complications and management. Moreover, we will suggest the risk and benefit of splenectomy or DP for optimal cytoreduction in AOC through a literature review.

\section{Anatomy}

Anatomical knowledge about the spleen and distal pancreas is imperative for better outcomes after splenectomy and DP. The spleen is located in the left upper quadrant of the human body, and the left diaphragm roofs its superior surface. It is protected by the left $9^{\text {th }}, 10^{\text {th }}$, and $11^{\text {th }}$ ribs, and suspended by multiple peritoneal reflections, including the splenophrenic, gastrosplenic, splenorenal, and splenocolic ligaments (Figure 1) (12). The stomach lies anterior to the spleen, and the splenic flexure of the colon and the left kidney are taken inferiorly and medially to it, respectively. Besides, the tail of the pancreas abuts on the splenic hilum.

In particular, the gastro-splenic ligament carries the short gastric arteries and veins in the superior aspect and the left gastro-epiploic artery and vein in the inferior aspect. The splenorenal ligament houses the splenic artery and vein, as well as the tail of the pancreas. The splenic artery is one of the branches of the celiac trunk, which is a tortuous vessel with multiple branches to the pancreas as it travels along its posterior aspect. On the other hand, the splenic vein leaves the splenic hilum and also travels posterior to the pancreas, joining with the inferior mesenteric vein to finally receive the superior mesenteric vein, forming the portal vein (Figure 2) (12).

\section{Operative procedure}

Although either laparoscopic or robot-assisted approach can perform splenectomy or DP, surgical treatment for most patients with AOC is usually laparotomy, and the proper methods also vary depending on the circumstances. The representative surgical techniques are as follows.

\section{Set-up}

A patient is placed either in a lithotomy position with keeping her arms straight along her sides or in a supine position with her arms extended. The most crucial point is to secure surgical visibility during surgery via adequate exposure of the left upper quadrant area. Since debulking 


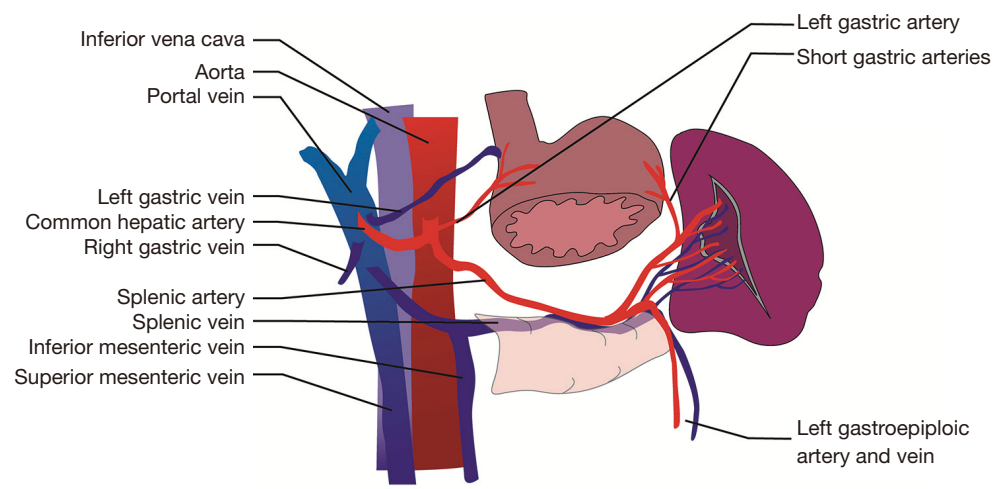

Figure 2 Clinical anatomy for performing splenectomy or distal pancreatectomy.

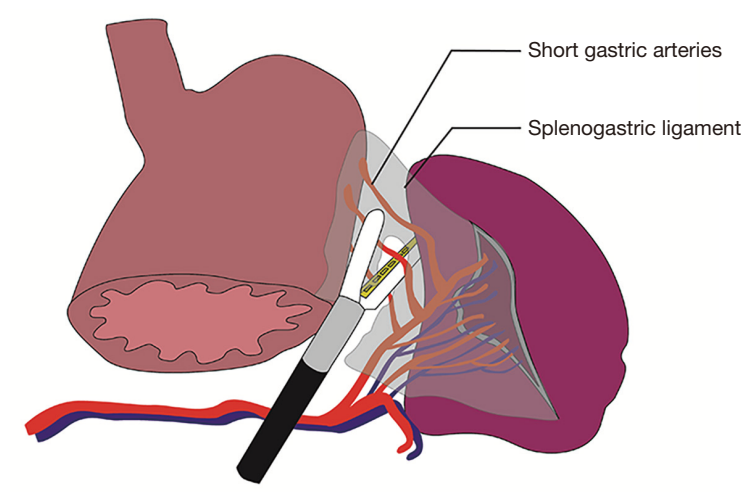

Figure 3 Resection of the gastrosplenic ligament, including the short gastric vessels.

surgery for AOC is performed through long midline incision to permit full exploration of the whole abdomen and pelvis, splenectomy or DP can be performed enough through the same incision by using self-retraining retractors without an additional incision in the left subcostal skin. Gynecologic oncologists stand either to the patient's right or between the patient's legs, and then the spleen and distal pancreas are carefully palpated to determine the extent of resection.

\section{Exposure of the spleen}

After the extent of resection is determined, the gastrosplenic ligament is opened by the end of resection of the gastro-colic ligament during debulking surgery, and the short gastric vessels are dissected carefully. In particular, vessel sealing or ultrasonic energy devices help reduce bleeding in this procedure (Figure 3).

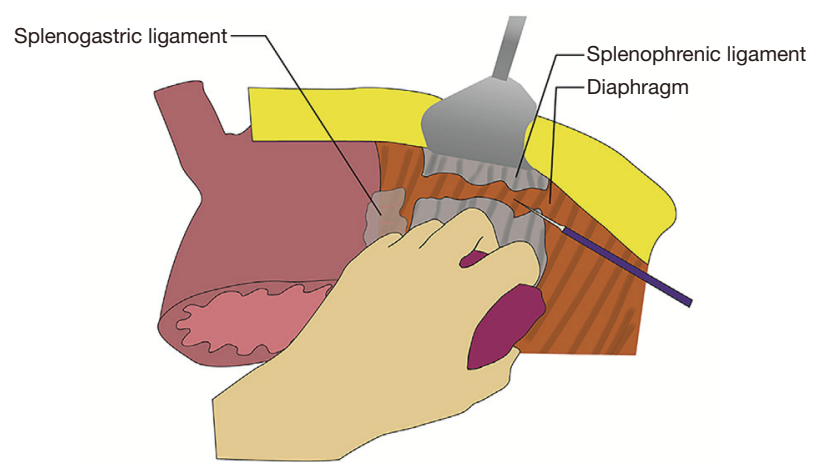

Figure 4 Mobilization of the spleen by dissecting the splenophrenic and splenorenal ligaments.

\section{Detachment of the splenic attachments}

The splenocolic ligament and splenic flexure are transected to get the spleen free from the colon. After that, the spleen is gently retracted medially to identify the peritoneal attachments. The attachments are divided with sharp dissection or monopolar cautery, proceeding from the inferior pole (the splenorenal ligament) to the superior pole (the splenophrenic ligament). Even small tears can result in much bleeding as the splenic capsule is very thin. Thus, precise and careful handling is required during this procedure (Figure 4).

\section{Removal of the spleen and distal pancreas}

After the spleen is fully mobilized, the splenic artery and vein are identified and ligated separately (Figure 5). However, the risk of significant bleeding increases when large tumors are located in the splenic hilum or extended 
to the splenic capsule. In that case, the proximal part of the splenic vessels toward the head of the pancreas should be ligated, and sometimes en bloc resection of the spleen and distal pancreas should be considered (12). For this procedure, a linear cutting stapler can be used to separate the spleen from the tail of the pancreas during splenectomy or to remove the pancreatic tail with the spleen together during en bloc resection. Even among general surgeons, DP with stapler has become famous for its simplicity and safety compared with the conventional hand-sewn closure (13). The pancreatic thickness usually determines the choice of a cartridge, but gold cartridges (closed staple height: $1.8 \mathrm{~mm}$ ) are mainly used for the standard dissection

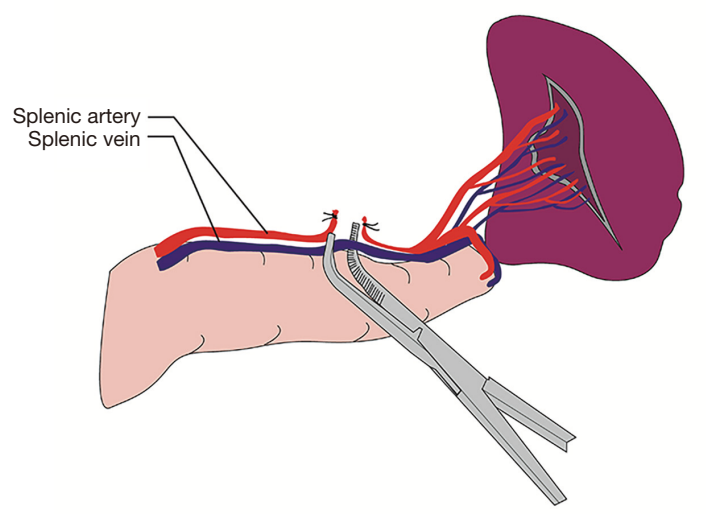

Figure 5 Identification and ligation of the splenic artery and vein.

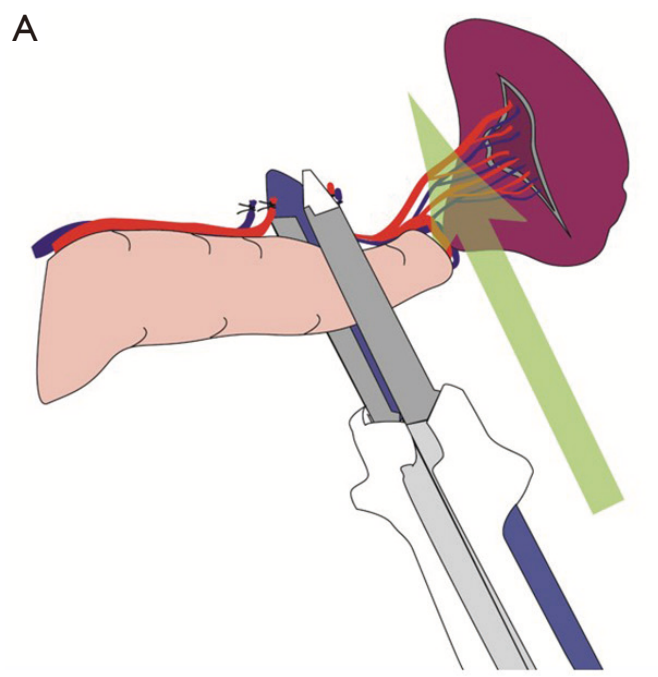

line of the pancreas with normal texture (14). After the cut section of the pancreas is inspected after DP, suture reinforcement on the cut section is performed with 2-0 or 3-0 prolene (Figure 6). After that, the surgical field is irrigated with warm saline and inspected for hemostasis. A drain is inserted around the site of splenectomy or DP for evaluating postoperative pancreatic fistula (POPF).

\section{Complications}

\section{Hemorrbage}

The incidence of hemorrhage is about 2.4\% (15). Bleeding can occur during exfoliating adhesions, tearing of the splenic capsule, loosening, or injuring the vessels around the spleen or the pancreas. Postoperative hemorrhage is accompanied by symptoms such as abdominal distension or pain, left shoulder pain, tachycardia, and hypotension with falling serum levels of hemoglobin. The splenic bed, the short gastric and splenic vessels, and the pancreatic tail are known as the common sites of hemorrhage (16).

\section{Infection}

Overwhelming post-splenectomy infection (OPSI) is the most common fatal complication after splenectomy, which represents pneumonia, bacteremia, and meningitis (17). The overall incidence and mortality of OPSI are $3.2-3.5 \%$

\section{B}

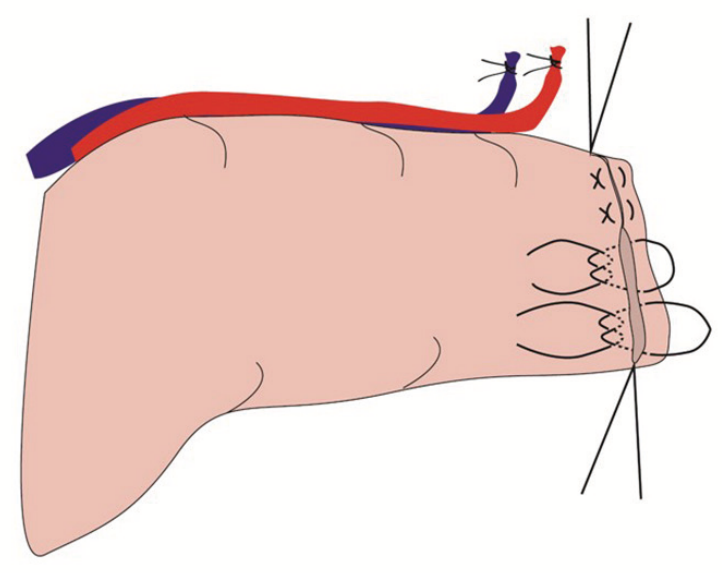

Figure 6 Splenectomy or distal pancreatectomy: (A) removal of the tail of the pancreas with linear cutting staplers, and the arrow indicate the resection line for splenectomy; (B) suture reinforcement on the cut section of the pancreas with 2-0 or 3-0 prolene after distal pancreatectomy. 
and $40-50 \%$ in patients with OPSI, of which the risk may increase in those with thalassemia major and sickle cell disease. Polysaccharide-encapsulated organisms, including Streptococcus pneumoniae, Neisseria meningitidis, and Haemophilus influenzae, typically cause OPSI (18).

In general, these organisms are bound in several types of antibodies and complement components in preparation for phagocytosis by macrophages in the spleen. However, the digestion by splenic macrophages is no longer possible in patients who underwent splenectomy. Moreover, they show subnormal levels of immunoglobulin $\mathrm{M}$, and mononuclear cells in their peripheral blood exhibit a suppressed immunoglobulin response (19).

Moreover, other factors involved in the immune response, such as properdin, tuftsin, and opsonins produced in the spleen, exhibit decreased serum levels after splenectomy. These factors are known to initiate the alternative pathway of complement activation. This increases the destruction of bacteria and foreign cells and enhances the phagocytic activity of mononuclear phagocytes and polymorphonuclear leukocytes (20).

\section{Injury to adjacent organs}

The stomach may be damaged by thermal injury during the dissection of the short gastric vessels or by mechanical trauma if not adequately decompressed with a nasogastric tube. This injury can cause gastric flatulence or fistula by necrosis of the gastric wall with abdominal distension (16).

Injury to the colon occurs rarely, but it can happen during mobilizing the splenic flexure of the colon, cautery injury, or direct trauma by electrosurgical devices (21).

\section{Postoperative pancreatic fistula (POPF)}

POPF is one of the most frequent complications after DP, which is sometimes regarded as a severe event that may increase hospitalization and relevant costs. People define POPF as an abnormal communication between the pancreatic ductal epithelium and another epithelial surface containing pancreas-derived, enzyme-rich fluid. Clinically, POPF represents a parenchymal leakage not directly related to an anastomosis such as one originating from the raw pancreatic surface due to failure of healing of the resected and sealed area in the pancreas.

After DP, POPF may be diagnosed based on clinically or biochemically suspicious findings. The common symptoms related to POPF may be leukocytosis, abdominal pain, and color change of drain fluid, showing a sinister appearance that may vary from a dark brown to greenish liquid by suspicious leakage from a bilioenteric anastomosis to milky to clear water after DP. Although leukocytosis is common after splenectomy, and as an isolated finding may not be related to infection (22), it may require a CT scan to evaluate fluid collection in the upper abdomen. Percutaneous drainage should be placed to avoid the worsening of POPF and subsequent abscess formation (23). Moreover, upper abdominal pain and distension, impaired bowel function, fever $>38^{\circ} \mathrm{C}$ can be combined.

In general, the following criteria according to International Study Group of Pancreatic Fistula (ISGPF) is used for the diagnosis of POPF: output via an operatively placed drain of any measurable volume of drain fluid on or after postoperative day 3; an amylase content more than three times the upper normal serum value (24).

Furthermore, ISGPF suggests the three-level grading system for POPF as follows. First, grade A POPF is the most common as transient fistula. It needs little change in management due to no clinical illness. Thus, most patients are fed orally and remain clinically well, and medical treatment, including total parenteral nutrition (TPN), antibiotics, and somatostatin analogs, is not required. Since imaging studies show no peri-pancreatic fluid collection, it can be resolved by the slow removal of the placed drains. Second, grade B POPF needs adequate medical treatments. For supportive care, nothing by mouth (NPO), partial or total parenteral nutrition, antibiotics, and somatostatin analogs are sometimes required, and repositioning of the drains should be performed by computed tomography or ultrasonography if they do not function to drain the fistula fully. However, most patients with grade B POPF can be discharged with drains in situ and observed in the outpatient setting. Third, grade C POPF requires aggressive management using NPO, TPN, intravenous antibiotics, and somatostatin analogs. Most patients with grade $\mathrm{C}$ POPF need extended hospitalization with a prolonged delay in hospital discharge and sometimes monitoring in an intensive care unit setting. Imaging studies usually show a worrisome, peripancreatic fluid collection, which requires percutaneous drainage. If deteriorating signs such as sepsis and organ dysfunction develop, reoperation should be considered. The prompt decision of reoperation can reduce postoperative morbidity and mortality for the following reasons: repair of the leakage site with extensive peripancreatic drainage; conversion to alternative means of pancreatic-enteric anastomosis (for example, conversion 
Table 1 International Study Group of Pancreatic Fistula (ISGPF) grading system for postoperative pancreatic fistula (POPF)

\begin{tabular}{lccc}
\hline Grade & A & B & C \\
\hline Clinical conditions & Well & Often well & III-looking or bad \\
Specific treatment* & No & No or yes & Yes \\
US/CT (if obtained) & Negative & Negative or positive & Positive \\
Persistent drainage after 3 weeks $^{\dagger}$ & No & Usually yes & No \\
Need of reoperation & No & No & Yes \\
Death related to POPF & No & Yes & Yessibly yes \\
Sings of infections & No & No & Yes \\
Sepsis & No & No or yes & No or yes \\
Readmission & No & Nath
\end{tabular}

${ }^{*}$, partial or total parenteral nutrition, antibiotics, somatostatin analogue, and/or minimal invasive drainage; ${ }^{\dagger}$, with or without a daring in situ.

of pancreaticojejunostomy to pancreaticogastrostomy); completion of pancreatectomy (Table 1).

Recently, endoscopic drainage for persistent pancreatic fistula can be performed by a trans-papillary or trans-mural approach, called as "endoscopic trans-papillary or transmural drainage of pancreatic collection or leaks" (25). This endoscopic treatment aims to create a connection between the pseudocyst cavity and the gastrointestinal lumen (26), and it is a safe and effective procedure showing a high success rate of $85 \%$ and low morbidity of $6 \%(27,28)$. During this procedure, a stent of 5 to $7 \mathrm{~F}$ (up to $10 \mathrm{~F}$ ) in diameter is inserted over the wire. The duration of stenting depends on the regression period of pancreatic fistula. In general, stents should be left in place for a longer-term over two months because their removal within two months has been reported to be related to a higher incidence of recurrence (29). Moreover, stents should be exchanged every six to eight weeks for as long as the pancreatic fistula remained unresolved (30).

\section{Others}

Thromboembolism after splenectomy can occur in up to $10 \%$ because splenectomy is associated with hypercoagulable states such as an increased level of prothrombotic factors $(31,32)$. In particular, reactive thrombocytosis may occur in $75-82 \%$, with a peak reached at 7-20 days after splenectomy, which can lead to thromboembolism by platelet hyper aggregation (33). Moreover, subphrenic abscess or cellulitis, atelectasis, pneumonia, and pleural effusion can also occur after splenectomy with or without DP (17).

\section{Management}

\section{Antibiotic prophylaxis}

In the early postoperative period, perioperative antibiotic prophylaxis is enough with low-dose amoxicillin until oral diet, and then can be switched to long-term oral antibiotic prophylaxis (34). Even though there is no consensus on the duration of treatment with antibiotics, and some guidelines recommend lifelong treatment regarding the persistent risk of sepsis, in particular, for immunosuppressed asplenic patients (35). At least, asplenic patients with solid tumors, including ovarian cancer, should be provided with a 5-day course of antibiotics, educated to seek immediate medical attention if symptoms or signs of infection develop (36).

\section{Vaccine prophylaxis}

For preventing OPSI, the standard care after splenectomy is immunization with polyvalent pneumococcal vaccine (PPV23), Haemophilus influenzae type b conjugate, and meningococcal polysaccharide vaccine within two weeks of splenectomy if you do not administer these vaccines before surgery. In particular, PPV23 is composed of a purified preparation of pneumococcal capsular polysaccharide antigens of 23 types of Streptococcus pneumoniae (25 mg each) that cause $88 \%$ of bacteremic pneumococcal diseases (19).

\section{Postoperative pancreatic fistula}

Despite different methods of DP, such as hand-sewn suture and resection by a stapler device, a surgical effort may play 
a role in reducing the rate of POPF. In particular, suture reinforcement of the staple line has been demonstrated to decrease POPF when compared with no suture reinforcement $(0 \%$ vs. 39\%). We recommend that suture reinforcement of the pancreatic staple line to reduce the rate of POPF (37).

Patients with POPF can have a regular diet when they seem to be improved clinically or biochemically and do not show associated symptoms such as ileus. Although the use of somatostatin or an analog such as octreotide may contribute to a reduction of the volume of fistula output, it does not lead to fistula closure at an earlier time (23). Moreover, the routine use of prophylactic administration is still controversial. While a randomized controlled trial (RCT) showed that it reduced leakage of pancreatic juice effectively (38), the other RCT and meta-analysis failed to show a meaningful reduction of the rate of $\operatorname{POPF}(39,40)$. Regardless of the use of somatostatin or an analog such as octreotide, continued drainage with close monitoring for signs and symptoms of sepsis is needed, and simultaneous administration of broad-spectrum antibiotics is reasonable during continued drainage.

\section{Prophylaxis of thromboembolism}

Since the incidence of venous thromboembolism (VTE) is $12-29 \%$ after splenectomy (41), low-molecular-weight heparin should be administered, especially in patients with a high risk of VTE. If VTE or portal vein thrombosis is detected after splenectomy, high-dose heparin followed, after at least three weeks, by oral anticoagulant therapy is required, and aspirin can help reduce the risk (42).

\section{Literature review about splenectomy or distal pancreatectomy for advanced ovarian cancer}

For evaluating clinical outcomes of splenectomy with or without DP, we performed a literature search of the MEDLINE and EMBASE by using the following terms for ovarian cancer ("ovary" or "ovarian tumor" or "ovarian cancer" or "ovarian carcinoma"), splenectomy ("spleen" or "splenic" or "splenectomy") and DP ("pancreas" or "pancreatic" or "pancreatectomy"). When we included relevant studies published since 2000, we found 13 related studies. We divided the followings into two groups: Frist, ten studies where splenectomy was performed during cytoreductive surgery without preoperative consideration of DP (4,8-11,43-47); second, three studies where splenectomy was performed during cytoreductive surgery with preoperative consideration of DP $(6,23,48)$.

In ten studies where splenectomy was performed during cytoreductive surgery without preoperative consideration of DP, the rate of DP was $2.7-33.3 \%$, and no visible tumor and residual tumor $<1 \mathrm{~cm}$ were obtained in $42.4-100 \%$ and $81.7-$ $100 \%$ of patients, respectively, except one study where the information about the size of residual tumor was absent in about $51 \%$ (11). The median value of hospitalization ranged from 6 to 14 days, and POPF was observed in $2.7-14.3 \%$.

In three studies where splenectomy was performed during cytoreductive surgery with preoperative consideration of DP, the success rate of optimal cytoreductive surgery was similar (no visible tumor, 53-100\%; residual tumor $<1 \mathrm{~cm}$, 94-100\%). However, the median value of hospitalization and the risk of POPF were slightly increased (9-33 days; 5.6-24\%). These findings suggest that the effort for preserving the tail of the pancreas might contribute to a reduction of hospitalization and the risk of POPF despite the minimal impact of DP on the success rate of optimal cytoreductive surgery (Table 2).

\section{Conclusions}

Splenectomy or DP is required for optimal cytoreduction in AOC. Knowledge about the relevant anatomy and operative procedure is necessary for gynecologic oncologists to perform this surgery safely. Moreover, a good understanding of complications and management will help build clinical experience for them. 
Table 2 Literature review about splenectomy with or without distal pancreatectomy for advanced or recurrent ovarian cancer

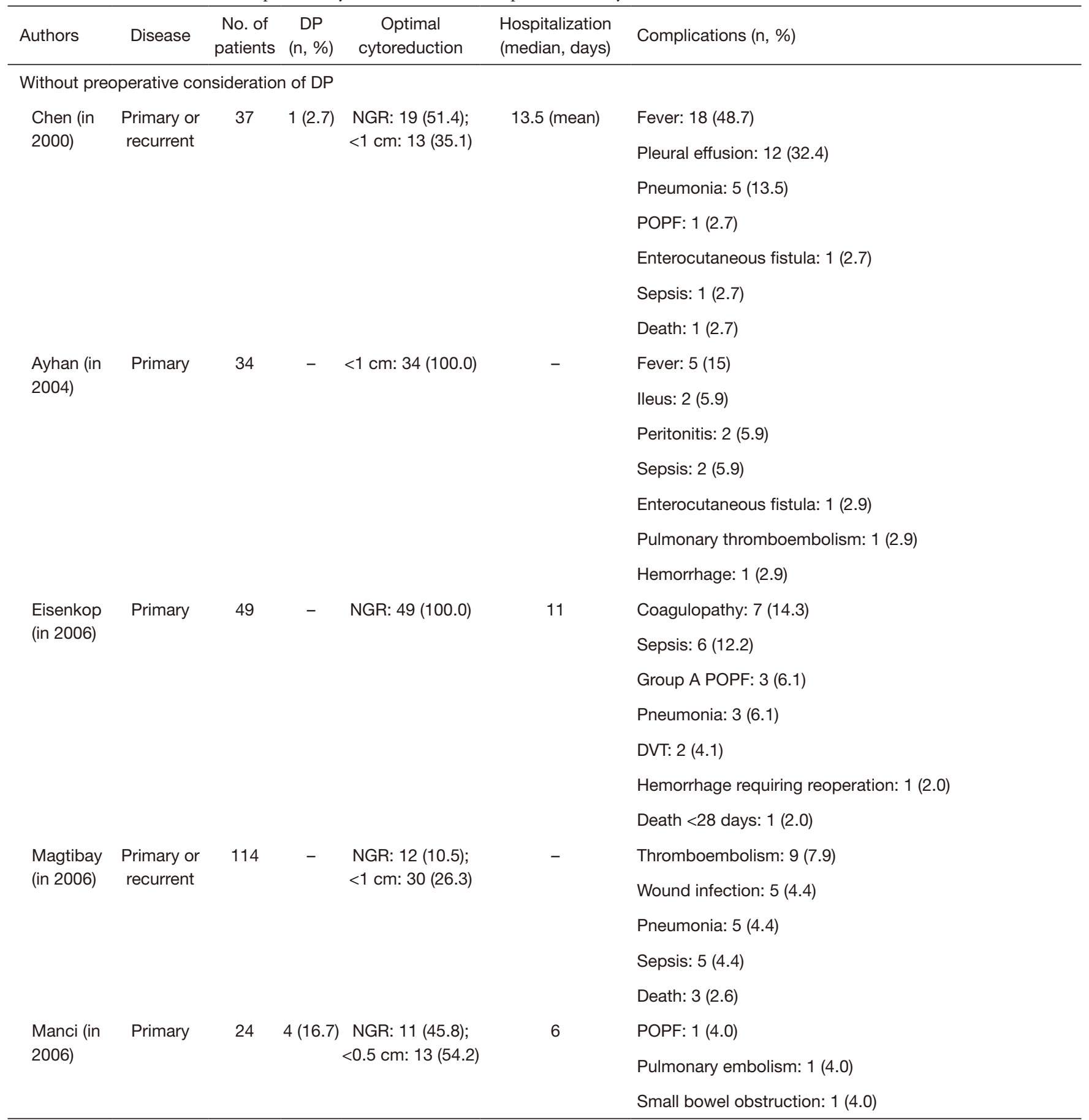

Table 2 (continued) 
Table 2 (continued)

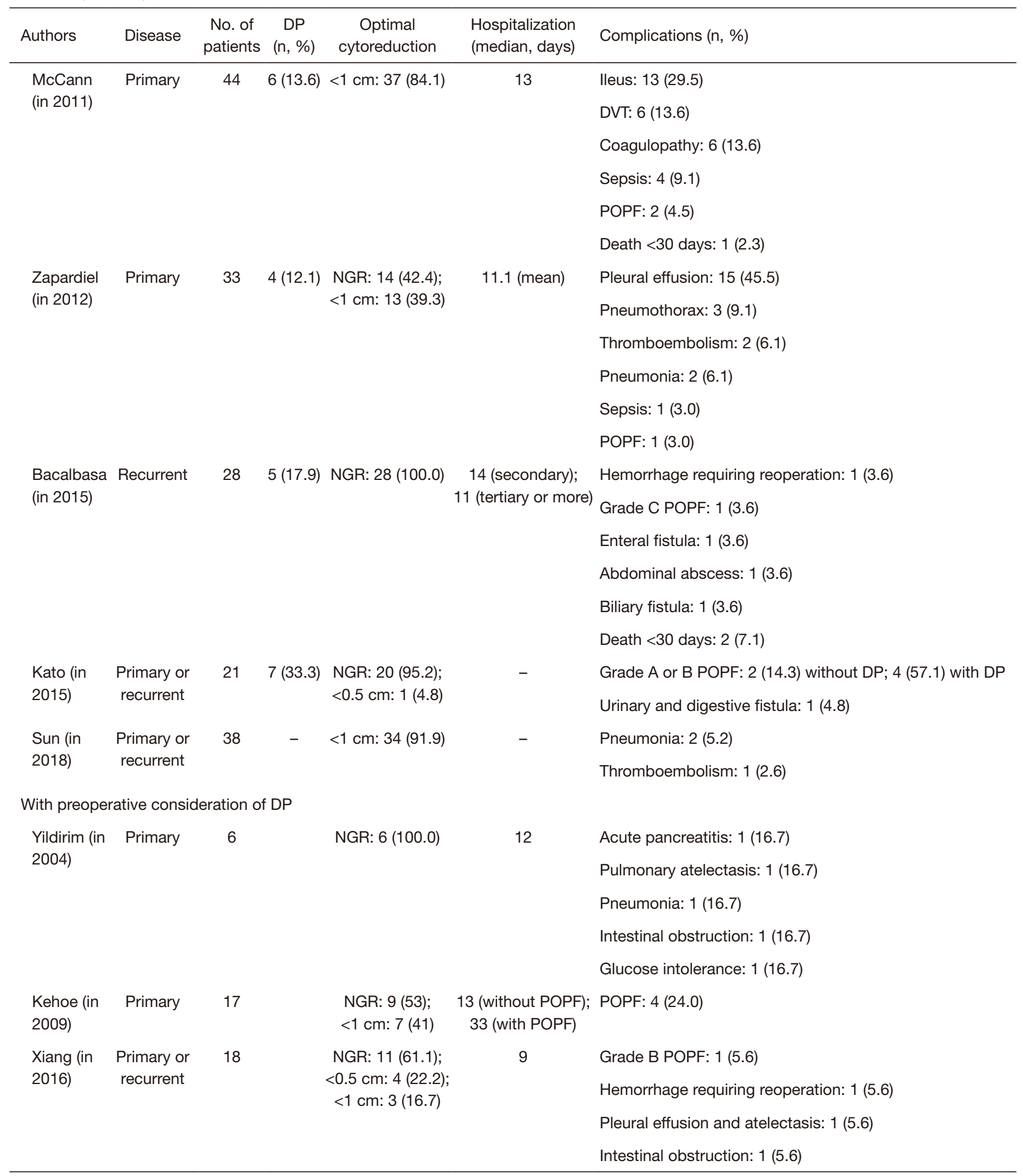

DP, distal pancreatectomy; DVT, deep vein thrombosis; NGR, no gross residual; POPF, postoperative pancreatic fistula. 


\section{Acknowledgments}

Funding: Grants from Seoul National University supported this study (No. 800-20170249, 800-20180201, 80020190437), and a grant of the Korea Health Technology R\&D Project through the Korea Health Industry Development Institute (KHIDI), funded by the Ministry of Health \& Welfare, the Republic of Korea (No. HI19C0664).

\section{Footnote}

Provenance and Peer Review: This article was commissioned by the Guest Editors (Sang Yoon Park, Jae Weon Kim) for the series "Ultra-Radical Surgery in Ovarian Cancer: Surgical Techniques for Gynecologic Oncologist" published in Gland Surgery. The article was sent for external peer review organized by the Guest Editors and the editorial office.

Conflicts of Interest: All authors have completed the ICMJE uniform disclosure form (available at http://dx.doi. org/10.21037/gs-2019-ursoc-09). The series "UltraRadical Surgery in Ovarian Cancer: Surgical Techniques for Gynecologic Oncologist" was commissioned by the editorial office without any funding or sponsorship. HSK serves as an unpaid editorial board member of Gland Surgery from August, 2019 to July, 2021. The authors have no other conflicts of interest to declare.

Ethical Statement: The authors are accountable for all aspects of the work in ensuring that questions related to the accuracy or integrity of any part of the work are appropriately investigated and resolved.

Open Access Statement: This is an Open Access article distributed in accordance with the Creative Commons Attribution-NonCommercial-NoDerivs 4.0 International License (CC BY-NC-ND 4.0), which permits the noncommercial replication and distribution of the article with the strict proviso that no changes or edits are made and the original work is properly cited (including links to both the formal publication through the relevant DOI and the license). See: https://creativecommons.org/licenses/by-nc-nd/4.0/.

\section{References}

1. Shih KK, Chi DS. Maximal cytoreductive effort in epithelial ovarian cancer surgery. J Gynecol Oncol 2010;21:75-80.

2. Park SJ, Kim J, Kim SN, et al. Practice patterns of surgery for advanced ovarian cancer: analysis from international surveys. Jpn J Clin Oncol 2019;49:137-45.

3. Koh YS, Kim JC, Cho CK. Splenectomy for solitary splenic metastasis of ovarian cancer. BMC Cancer 2004;4:96.

4. Sun H, Bi X, Cao D, et al. Splenectomy during cytoreductive surgery in epithelial ovarian cancer. Cancer Manag Res 2018;10:3473-82.

5. Otrock ZK, Seoud MA, Khalifeh MJ, et al. Laparoscopic splenectomy for isolated parenchymal splenic metastasis of ovarian cancer. Int J Gynecol Cancer 2006;16:1933-5 .

6. Yildirim Y, Sanci M. The feasibility and morbidity of distal pancreatectomy in extensive cytoreductive surgery for advanced epithelial ovarian cancer. Arch Gynecol Obstet 2005;272:31-4.

7. Guidozzi F, Ball JH. Extensive primary cytoreductive surgery for advanced epithelial ovarian cancer. Gynecol Oncol 1994;53:326-30.

8. Zapardiel I, Peiretti M, Zanagnolo V, et al. Splenectomy as part of primary cytoreductive surgery for advanced ovarian cancer: a retrospective cohort study. Int J Gynecol Cancer 2012;22:968-73.

9. Kato K, Tate S, Nishikimi K, et al. Management of pancreatic fistulas after a splenectomy as part of cytoreductive surgery for ovarian cancer. Int J Gynecol Cancer 2013;23:1506-11.

10. McCann CK, Growdon WB, Munro EG, et al. Prognostic significance of splenectomy as part of initial cytoreductive surgery in ovarian cancer. Ann Surg Oncol 2011;18:2912-8.

11. Magtibay PM, Adams PB, Silverman MB, et al. Splenectomy as part of cytoreductive surgery in ovarian cancer. Gynecol Oncol 2006;102:369-74.

12. Ramirez PT, Dos Reis R. Splenectomy in patients with advanced or recurrent ovarian cancer: open and laparoscopic surgical techniques and clinical outcomes. Gynecol Oncol 2007;104:29-32.

13. Kim H, Jang JY, Son DH, et al. Optimal Stapler Cartridge Selection According to the Thickness of the Pancreas in Distal Pancreatectomy. Medicine 2016;95:e4441-6.

14. Okano K, Kakinoki K, Yachida S, et al. A simple and safe pancreas transection using a stapling device for a distal pancreatectomy. J Hepatobiliary Pancreat Surg 2008;15:353-8.

15. Winslow ER, Brunt LM. Perioperative outcomes of 
laparoscopic versus open splenectomy: a meta-analysis with an emphasis on complications. Surgery 2003;134:64753; discussion 654-45.

16. Qu Y, Ren S, Li C, et al. Management of postoperative complications following splenectomy. Int Surg 2013;98:55-60.

17. Horowitz J, Smith JL, Weber TK, et al. Postoperative complications after splenectomy for hematologic malignancies. Ann Surg 1996;223:290-6.

18. Spelman D, Buttery J, Daley A, et al. Guidelines for the prevention of sepsis in asplenic and hyposplenic patients. Intern Med J 2008;38:349-56.

19. Shatz DV, Schinsky MF, Pais LB, et al. Immune responses of splenectomized trauma patients to the 23 -valent pneumococcal polysaccharide vaccine at 1 versus 7 versus 14 days after splenectomy. J Trauma 1998;44:760-5; discussion 765-766.

20. Kirkineska L, Perifanis V, Vasiliadis T. Functional hyposplenism. Hippokratia 2014;18:7-11.

21. Bhandarkar DS, Katara AN, Mittal G, et al. Prevention and management of complications of laparoscopic splenectomy. Indian J Surg 2011;73:324-30.

22. Bidus MA, Krivak TC, Howard R, et al. Hematologic changes after splenectomy for cytoreduction: implications for predicting infection and effects on chemotherapy. Int J Gynecol Cancer 2006;16:1957-62.

23. Kehoe SM, Eisenhauer EL, Abu-Rustum NR, et al. Incidence and management of pancreatic leaks after splenectomy with distal pancreatectomy performed during primary cytoreductive surgery for advanced ovarian, peritoneal, and fallopian tube cancer. Gynecol Oncol 2009; 112:496-500.

24. Bassi C, Dervenis C, Butturini G, et al. Postoperative pancreatic fistula: an international study group (ISGPF) definition. Surgery 2005;138:8-13.

25. Mutignani M, Dokas S, Tringali A, et al. Pancreatic Leaks and Fistulae: An Endoscopy-Oriented Classification. Dig Dis Sci 2017;62:2648-57.

26. Baron TH, Thaggard WG, Morgan DE, et al. Endoscopic therapy for organized pancreatic necrosis. Gastroenterology 1996;111:755-64.

27. Seicean A, Vultur S. Endoscopic therapy in chronic pancreatitis: current perspectives. Clin Exp Gastroenterol 2014;8:1-11.

28. Zerem E, Hauser G, Loga-Zec S, et al. Minimally invasive treatment of pancreatic pseudocysts. World J Gastroenterol 2015;21:6850-60.

29. Dumonceau JM, Delhaye M, Tringali A, et al. Endoscopic treatment of chronic pancreatitis: European Society of Gastrointestinal Endoscopy (ESGE) Clinical Guideline. Endoscopy 2012;44:784-800.

30. Catalano MF, Geenen JE, Schmalz MJ, et al. Treatment of pancreatic pseudocysts with ductal communication by transpapillary pancreatic duct endoprosthesis. Gastrointest Endosc 1995;42:214-8.

31. Mohren M, Markmann I, Dworschak U, et al. Thromboembolic complications after splenectomy for hematologic diseases. Am J Hematol 2004;76:143-7.

32. Feldman LS. Laparoscopic splenectomy: standardized approach. World J Surg 2011;35:1487-95.

33. Khan PN, Nair RJ, Olivares J, et al. Postsplenectomy reactive thrombocytosis. Proc (Bayl Univ Med Cent) 2009;22:9-12.

34. Buzelé R, Barbier L, Sauvanet A, et al. Medical complications following splenectomy. J Visc Surg 2016;153:277-86.

35. Di Sabatino A, Carsetti R, Corazza GR. Post-splenectomy and hyposplenic states. Lancet 2011;378:86-97.

36. Harji DP, Jaunoo S, Mistry P, et al. Immunoprophylaxis in asplenic patients. Int J Surg 2009;7:421-3.

37. Jimenez RE, Mavanur A, Macaulay WP. Staple line reinforcement reduces postoperative pancreatic stump leak after distal pancreatectomy. J Gastrointest Surg 2007;11:345-9.

38. Gouillat C, Chipponi J, Baulieux J, et al. Randomized controlled multicentre trial of somatostatin infusion after pancreaticoduodenectomy. Br J Surg 2001;88:1456-62.

39. Yeo CJ, Cameron JL, Lillemoe KD, et al. Does prophylactic octreotide decrease the rates of pancreatic fistula and other complications after pancreaticoduodenectomy? Results of a prospective randomized placebo-controlled trial. Ann Surg 2000;232:419-29.

40. Zeng Q, Zhang Q, Han S, et al. Efficacy of somatostatin and its analogues in prevention of postoperative complications after pancreaticoduodenectomy: a metaanalysis of randomized controlled trials. Pancreas 2008;36:18-25.

41. Ha LP, Arrendondo M. Fatal venous thromboembolism after splenectomy: pathogenesis and management. J Am Osteopath Assoc 2012;112:291-300.

42. Vecchio R, Cacciola E, Cacciola RR, et al. Portal vein thrombosis after laparoscopic and open splenectomy. J Laparoendosc Adv Surg Tech A 2011;21:71-5.

43. Chen LM, Leuchter RS, Lagasse LD, et al. Splenectomy and surgical cytoreduction for ovarian cancer. Gynecol 
Oncol 2000;77:362-8.

44. Ayhan A, Al RA, Baykal C, et al. The influence of splenic metastases on survival in FIGO stage IIIC epithelial ovarian cancer. Int J Gynecol Cancer 2004;14:51-6.

45. Eisenkop SM, Spirtos NM, Lin WC. Splenectomy in the context of primary cytoreductive operations for advanced epithelial ovarian cancer. Gynecol Oncol 2006;100:344-8.

46. Manci N, Bellati F, Muzii L, et al. Splenectomy during secondary cytoreduction for ovarian cancer disease

Cite this article as: Lee EJ, Park SJ, Kim HS. Splenectomy and distal pancreatectomy in advanced ovarian cancer. Gland Surg 2021;10(3):1218-1229. doi: 10.21037/gs-2019-ursoc-09 recurrence: surgical and survival data. Ann Surg Oncol 2006;13:1717-23.

47. Bacalbasa N, Balescu I, Dima S, et al. Splenectomy as Part of Cytoreductive Surgery in Recurrent Epithelial Ovarian Cancer. Anticancer Res 2015;35:5097-101.

48. Xiang L, Tu Y, He T, et al. Distal pancreatectomy with splenectomy for the management of splenic hilum metastasis in cytoreductive surgery of epithelial ovarian cancer. J Gynecol Oncol 2016;27:e62. 